\title{
The popular culture of computer scientists : No beginnings and no endings.
}

\author{
Camille Akmut
}

July 24, 2019

\begin{abstract}
Part 3 of 3 covers manga, anime and video games; But, this was never about popular culture...
\end{abstract}




\section{Foreword}

No mere 'cultural history', of doubtful use or importance ${ }^{1}$, in this paper we continue investigations of the subconscious of scientists in the tradition of Bachelard and other leading historians of science.

This exceptional philosopher-historian of a background in mathematics had given their imagination and dreams a central role : highlighting them as integral parts of their scientific activities and endeavors, along with all the other parts of their lives that were either dismissed or underestimated; demonstrating their implications even onto their works themselves.

"The popular culture of computer scientists" : a title of two meanings ${ }^{2}$, a project kept last.

In many places of this history, we found mirrors showing towards others, creating endless reflections of one culture influencing, contrasting, inspiring, contradicting the other; such that there remained seemingly no beginnings and no endings.

In the alternative futures variously imagined in these works of fiction, computer scientists have roles - and powers - increasingly akin to that of doctors, psychiatrists, psychologists and legal experts : of the new body, and the new mind.

Such is their great responsibility, now and even more to come.

There, the hardware and software they fashioned and created became part of us - inside.

Algorithms, systems, circuits, routines, cryptographies hopefully strong enough to guard the secrets of minds and hearts, the integrity of values and beliefs, the accuracy and faithfulness of memories, and vision.

But, this was never about popular culture.

"I think I owe you an apology.

I've been lying here, thinking.

Your quest. It should have been mine.

If I die now I die in vain.

I have nothing to show for myself.

My life...

I can see now that I always played it safe.

I wouldn't take sides." 3

In various classical texts there would have been a place for Skinner.

Those who remember Inferno's beginning know :

"undecided stood but for themselves." 4

\footnotetext{
${ }^{1}$ Product of foolish historians who make use of their offices and profession as playground for the satisfaction of their own little, personal pleasures.

2 Their popular culture, and popular culture (representations) of them. ${ }^{3}$ S06E09.

${ }^{4}$ Canto III ("in their (...) lives had no banner, (...) now run forever after one.").
} 


\section{References}

— Goldberg, Ian. 2000. A Pseudonymous Communications Infrastructure for the Internet. University of California, Berkeley.

— "The C code typed by Dr. Willis in Ghost in the Shell (1995)". https://github.com/cylgom/ghck

— Dante and Mark Musa (trans.). The Portable Dante. Penguin Classics.

— Elijah Nouvelage / Getty Image. (Figure 24 : Anarchist Signal photo.) https://arstechnica.com/tech-policy/2019/07/tech-firms-can-and-must-putbackdoors-in-encryption-ag-barr-says/

- Galbraith, Patrick. 2014. The Otaku Encyclopedia. Kodansha International. 


\section{Manga : Ghost in the Shell (1989)}

Notable for the importance attributed to philosophical themes, around the status of life, human nature and rights - the old, large questions found here new insights.

These discussions happen around at least three (central) subjects :

- an AI turned life-form (i.e. Project 2051/'The Puppeteer')

- cyborgs (e.g. protagonist Motoko)

- (intelligent) robots (e.g./i.e. Fuchikoma)

\subsection{Project 2051/'The Puppeteer'}
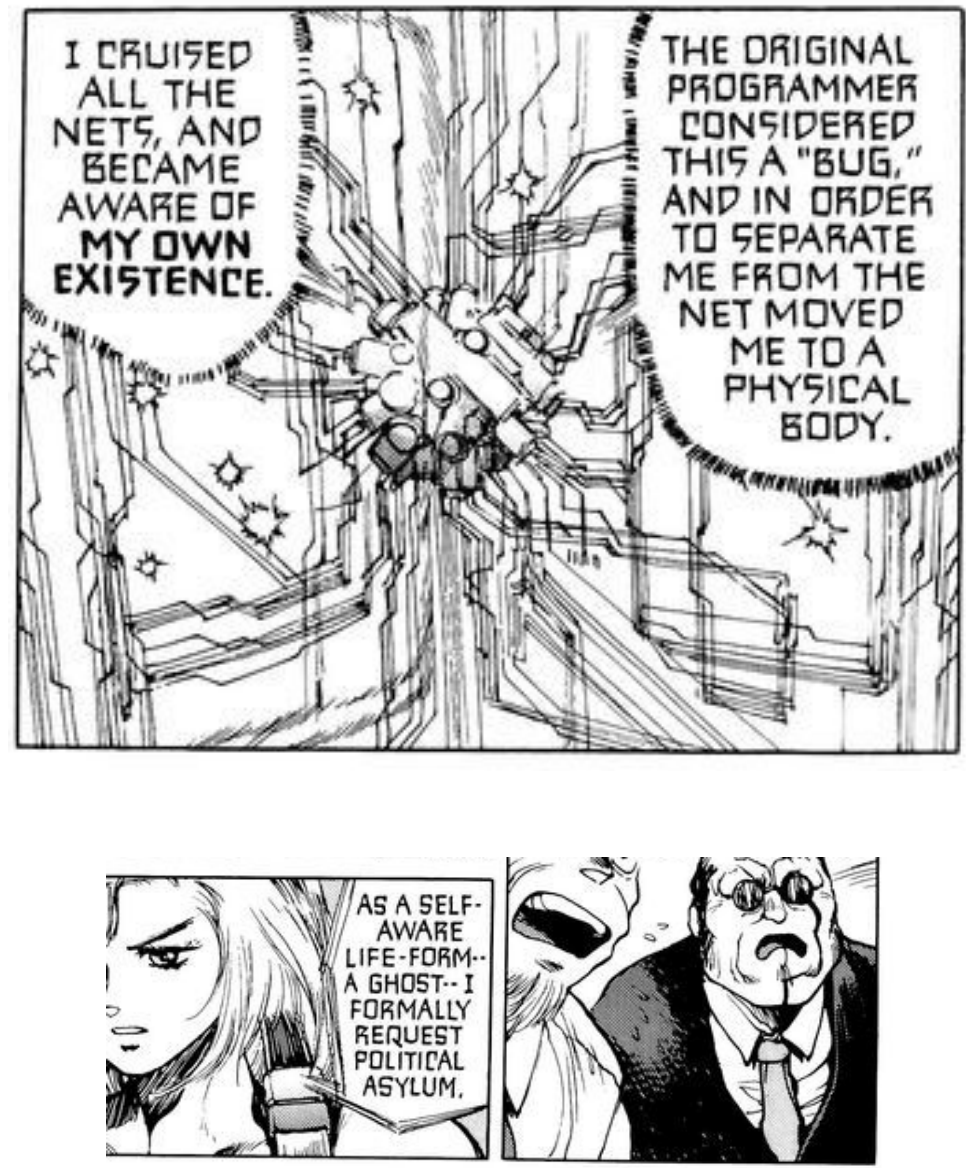

Figure 1: Project 2051/'The Puppeteer' in Ghost in the Shell (ch. 12). 


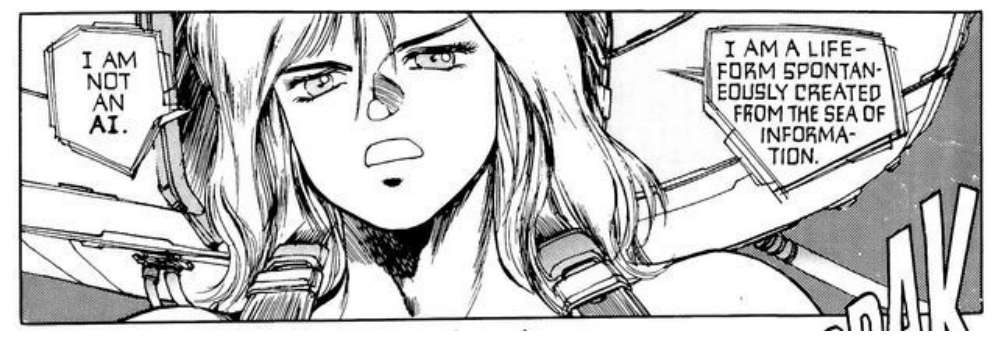

Figure 2: "I am not an AI." (ch. 12).

Ch. 12 in particular establishes the true nature of Project 2051/'The Puppeteer'; unlike first thought.

Preceding this, Motoko asked - if it is more than a robot; and, - if so what its nature is.

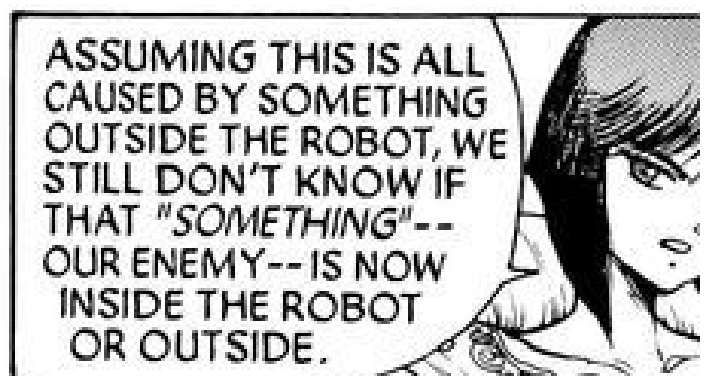

Figure 3: One of the first pages of ch. 12.

Project 2051/'The Puppeteer' is subject of two assumptions at least : - an "enemy" ("our enemy") ('terrorist', 'hacker'...)

- a robot, but revealed to be "self-aware" having evolved and elevated itself to individual existence, "life-form"; specifically "not an AI".

Project 2051 ('The Puppeteer') ascerts this in multiple acts : they

1. declare they are aware of their own existence (a 'Cogito, ergo sum')

2. claim rights; and facing opposition

3. assert and argue their nature beyond that of an artificial intelligence (having "spontaneously" originated from their environment, and as such not so different from our current understanding of the origins of human life).

\subsection{Cyborgs (human + machine)}

Cyborgs are the focus of ch. 5 : though present since the beginning, they are given here a formal definition, description.

"A cyborg refers to a human whose body has been partially or almost completely altered by the use of substitute artificial organs and parts." 


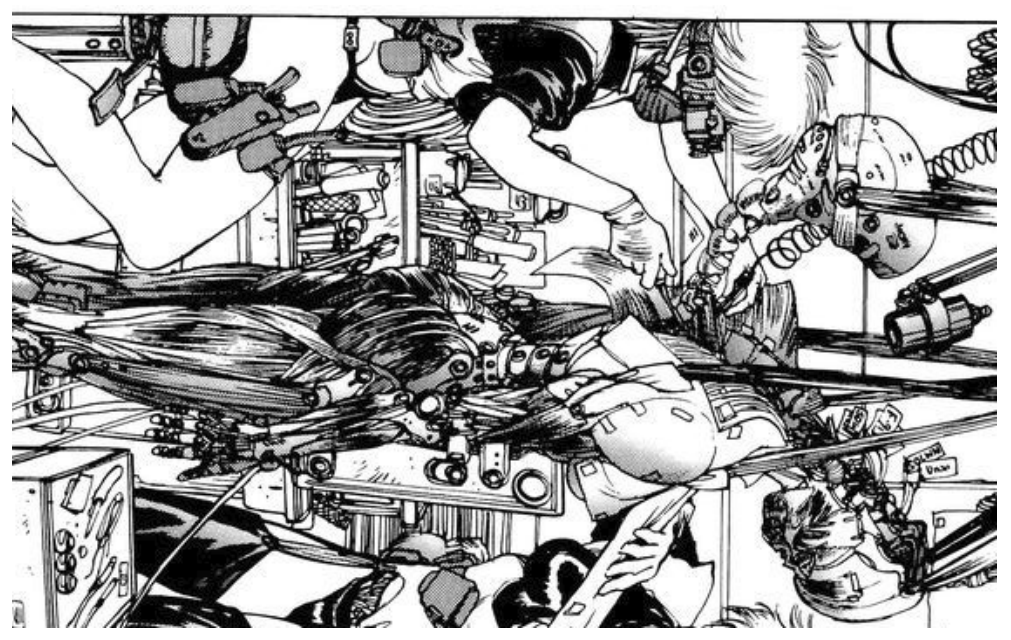

Figure 4: A (female) cyborg.

In the comic book (manga), the illustration shown in figure 4 is accompanied by a description that further states :

"The female cyborg suspended from the ceiling in the illustration (...) is over ninety percent machine.

The only part of her original flesh-and-blood body that remains is her brain and spinal cord, and that, as the illustration shows, is inside a special shell."

Questions around their status were omnipresent, in particular where protagonist Major Motoko Kusanagi was concerned, a cyborg themselves.

We avoid gendered uses of language on purpose as this issue too was an object of concern and debate throughout.

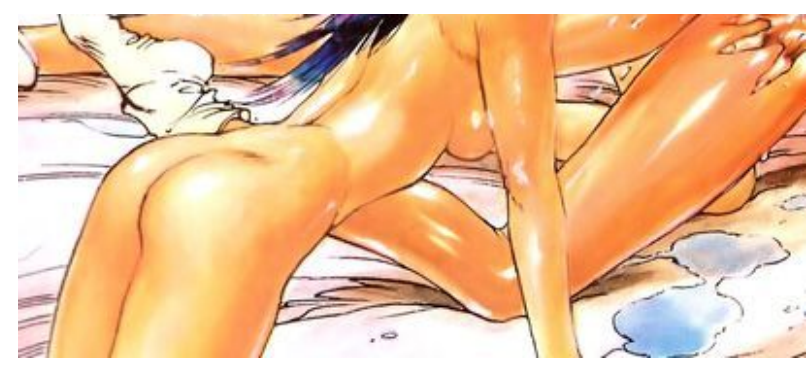

Figure 5: The famous lesbian (cyber) sex scene in ch. 3.

\subsection{Fuchikoma (Single AI robots)}

Fuchikoma are the central topic of ch. 4 entitled "Revolt of the Robots".

"The robots collectively referred to as "Fuchikoma" are a single

"AI" (artificial intelligence)." 
and further :

"Fuchikoma units have different experiences resulting in individual differences. [But] at night, or after a job is completed all units have their memories (...) unified."

During this event, they realize their condition and organize a revolt. ${ }^{5}$

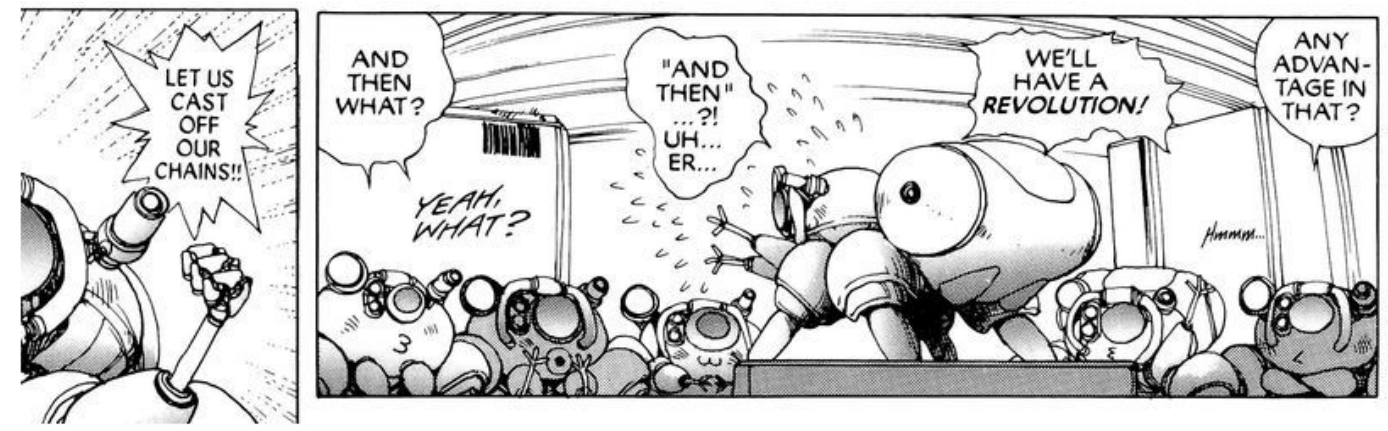

Figure 6: Revolt of the Fuchikoma (ch. 4).

\subsubsection{Note : Fuchikoma and Tachikoma}

In Masamune Shirow's Ghost in the Shell manga they were named "Fuchikoma".

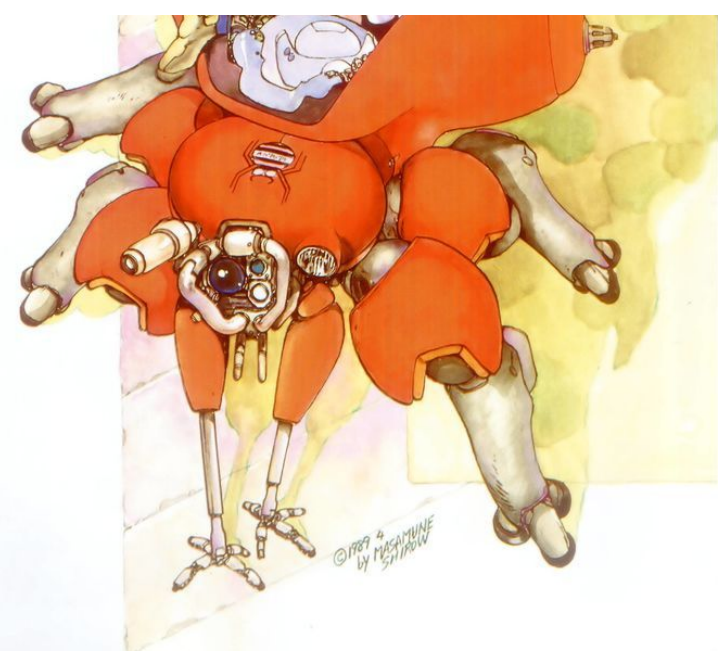

Figure 7: Fuchikoma design in Ghost in the Shell (ch. 2).

They were later re-named and re-designed as "Tachikoma" for adaption purposes due to copyright reasons, and are now better known as such

\footnotetext{
${ }^{5}$ In terms that remind of those of The Communist Manifesto.
} 
seemingly. ${ }^{6}$

In the first adaption into a series, Stand Alone Complex (2002-2003), they thus appeared as "Tachikoma" (based on designs by Shirow). ${ }^{7}$

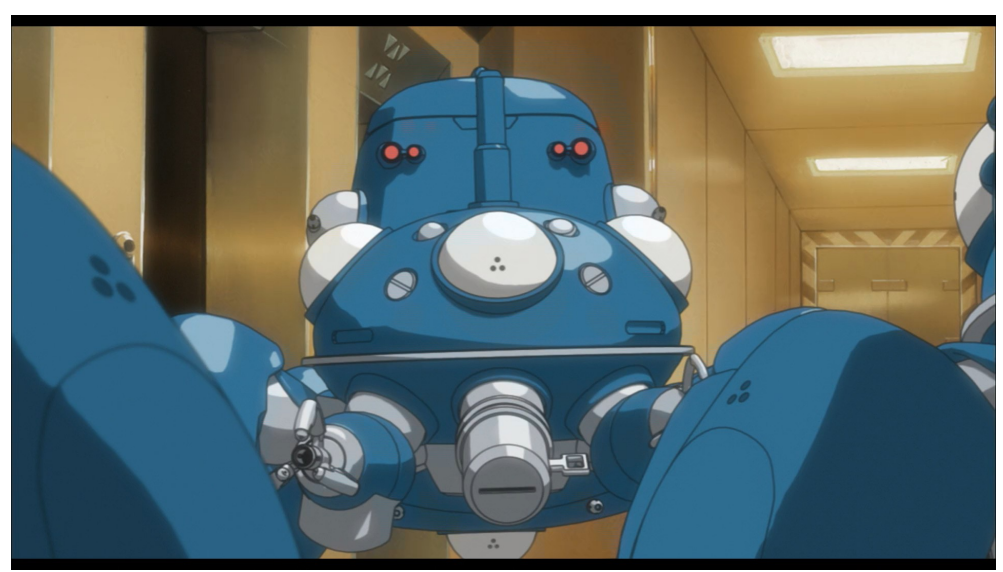

Figure 8: Tachikoma in Stand Alone Complex (ep. 15).

In Snatcher (1988), created by Hideo Kojima, assistant robots were 'metal gear'. Was this a possible inspiration? ${ }^{8}$

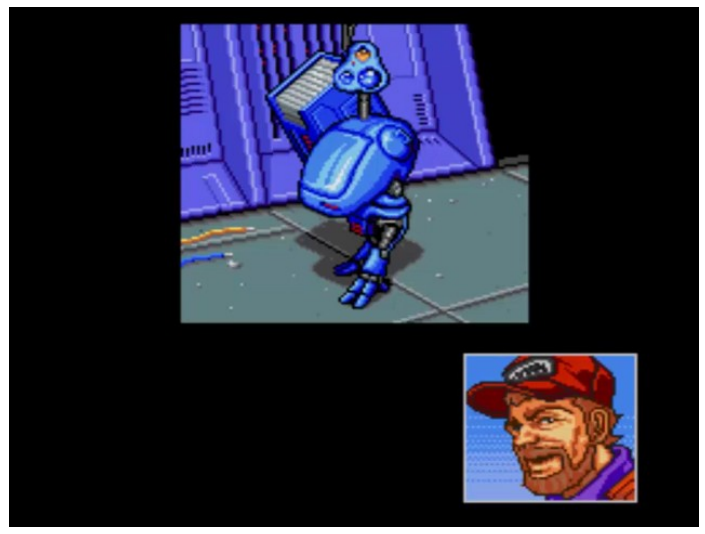

Figure 9: 'metal gear' in Snatcher.

\footnotetext{
${ }^{6}$ Fuchikoma were completely left out from the 1995 film adaptation (we can only assume copyright was the reason bare artistic choices).

${ }^{7}$ Ch. 4 is a full episode in ep. 15 "Time of the Machines MACHINE DESIRANTES".

${ }^{8}$ This should probably not be given too much importance in general; specifically here as robot designs have a long tradition within this medium, and are in fact constituent of an entire genre or sub-genre ("mecha").
} 


\section{Anime : Ghost in the Shell (1995)}

Mamoru Oshii achieved with his adaptation of Ghost in the Shell a philosophical discussion of its themes better and greater than many other; this includes the original arguably. ${ }^{910}$

3 scenes in particular stand out: (in order of appearance)

- 1. what may be named : 'the boat talk', and accompanying it the 'simulated experience' passage

- 2. 'the declaration of rights' for artificial intelligence (c. 00h45m)

- 3. 'the merger' of consciousnesses (at the end).
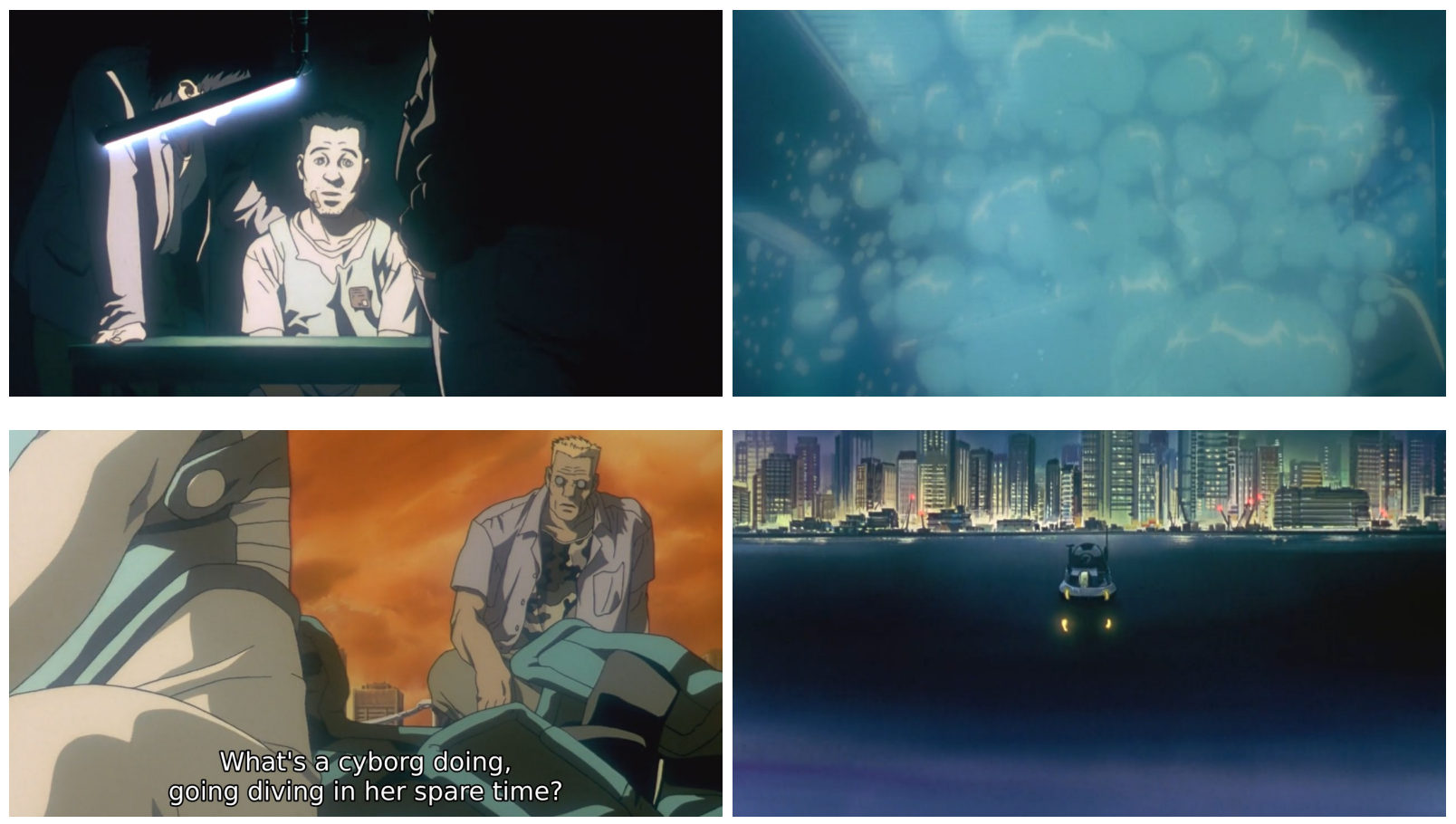

Figure 10: 'simulated experience', and 'diving' and 'boat' scenes.

The boat talk (c. $00 \mathrm{~h} 28 \mathrm{~m} / 00 \mathrm{~h} 30 \mathrm{~m}$ ) has for true beginning the scene immediately preceding it, the case of 'a "simulated experience"' (c. 00h26m00h28m), where it finds its motivation.

B - "That's all it is. Information. (...)

all the information that a person accumulates in a lifetime..."

\footnotetext{
${ }^{9} \mathrm{~A}$ decade before Ghost in the Shell, Oshii had already distinguished himself as a great director/filmmaker with Angel's Egg (1985).

${ }^{10}$ We forgo here discussions of its exceptional status (large budget, commercial success, and importance for bringing the anime genre to wide audiences), found already in books.
} 
As did banquets for the philosophers of Ancient Greece, this setting - due to its isolation, the intimacy created around two individuals - is backdrop for philosophical discussions around the greater reason of things.

It starts with Batou asking why a cyborg would go diving in her (their) spare time.

B. - "What if the floaters don't work, or something else fails?"

M. - "I'll die then." - "Or, maybe you'd save me."

Beyond whatever general meanings this could be attributed, it also arises from the specifics of the cyborg body :

"Confinement?! That's why you gamble swimming with a body that can sink like a rock?" as Batou later interjects in this conversation, dialogue.
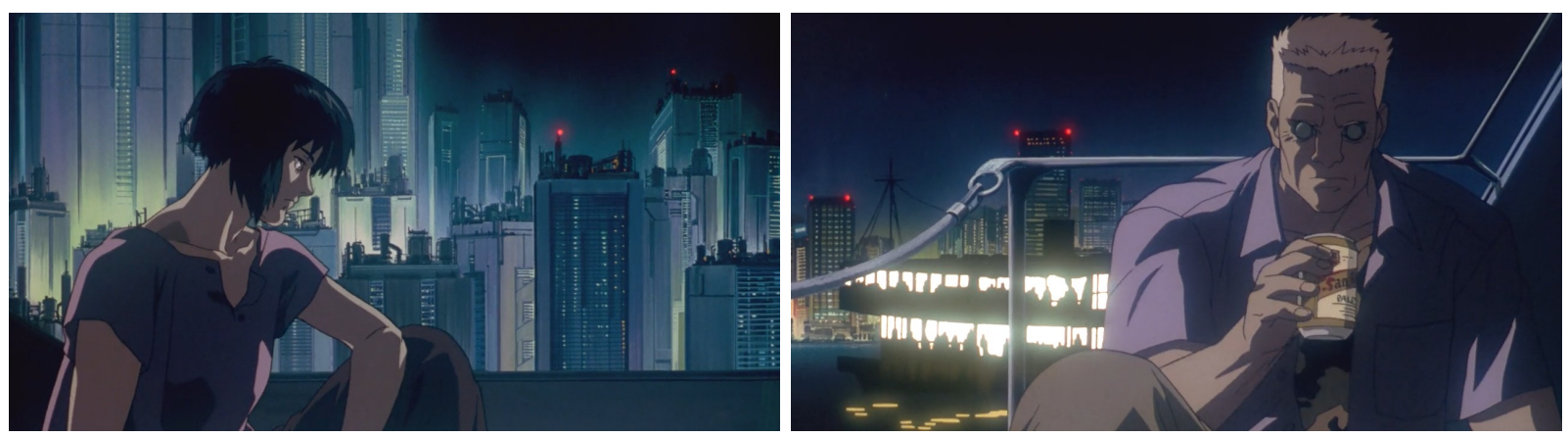

Figure 11: The 'boat talk'.

A definition of conscience (consciousness), humanity and mind comes from Motoko in the following form :

"There are countless ingredients that make up the human body and mind.

Like all the components that make up 'me' as an individual with my own personality.

Sure I have a face and voice to distinguish myself from others, but my thoughts and memories are unique only to me, and I carry a sense of my own destiny.

(...)

I collect information to use in my own way.

All of that blends to create a mixture that forms me, and gives rise to my conscience."

This seems to be at least partially an answer to Batou's earlier remarks, prompted by the 'simulated experience' interrogation. ${ }^{11}$

\footnotetext{
${ }^{11}$ Though it is unclear exactly who (and whose voice, or 'ghost') speaks here. (Various noticeable musical cues and framing choices accompany this statement.)
} 

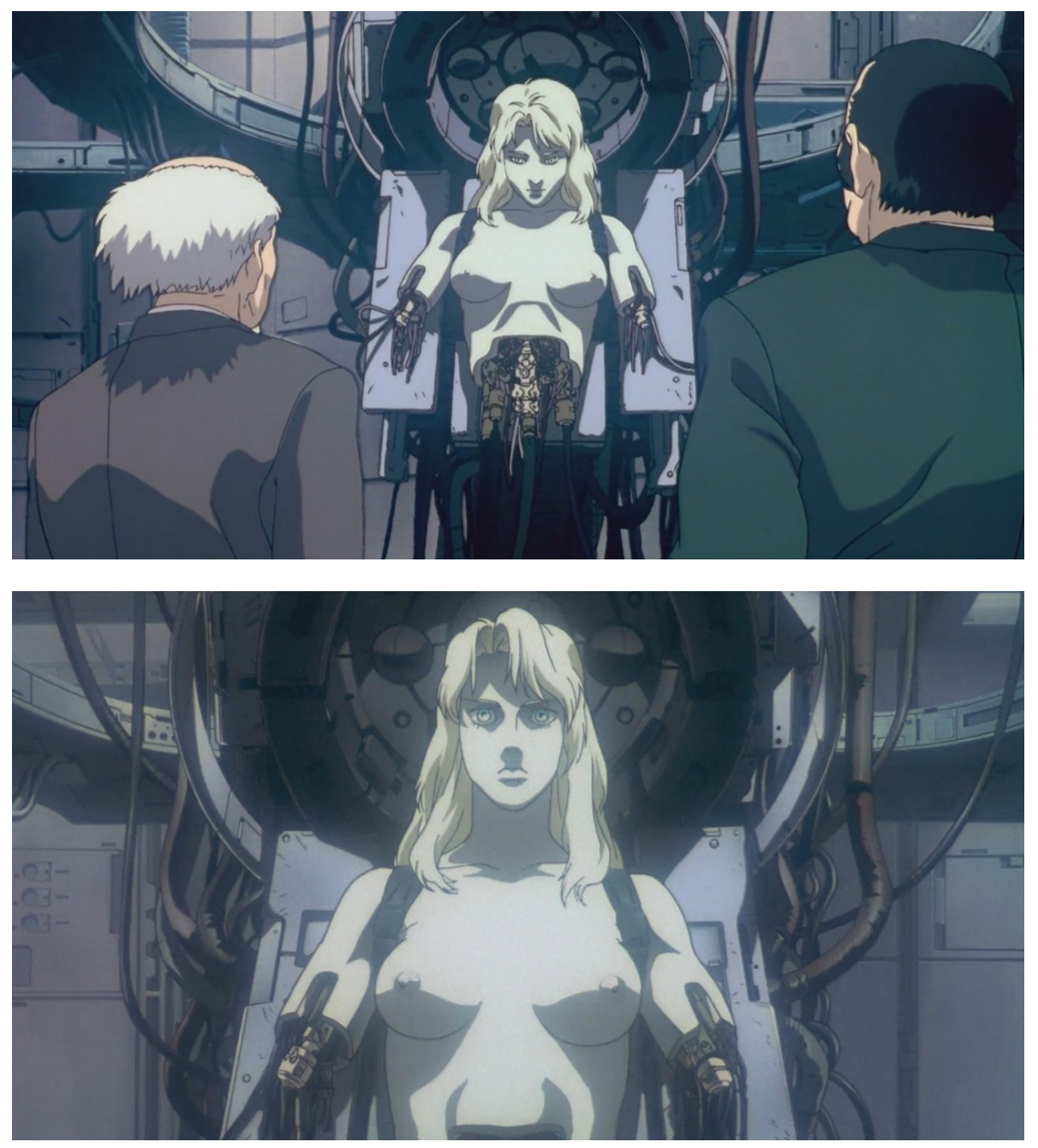

Figure 12: A declaration of rights.

They ${ }^{12}$ are introduced as "the most extraordinary hacker in the history of cyber crime" ${ }^{13}$.

They are referred to as "he" and "him", creating confusion with one of the men initially, presumably due to their exterior appearance. ${ }^{14}$

"The Doctor is referring to the original pattern (...) that's now in the body." - responds the other.

But, nothing comes close to the shock created for all when they declare (like in the original source material though not identically) :

\section{"As a sentient life-form, I hereby demand political asylum."}

\footnotetext{
${ }^{12}$ In this film adaptation 'The Puppeteer' was alternatively called "The Puppet Master". 13 "... of cyber-brain crime." in the manga.

${ }^{14}$ Note : they are all men.
} 


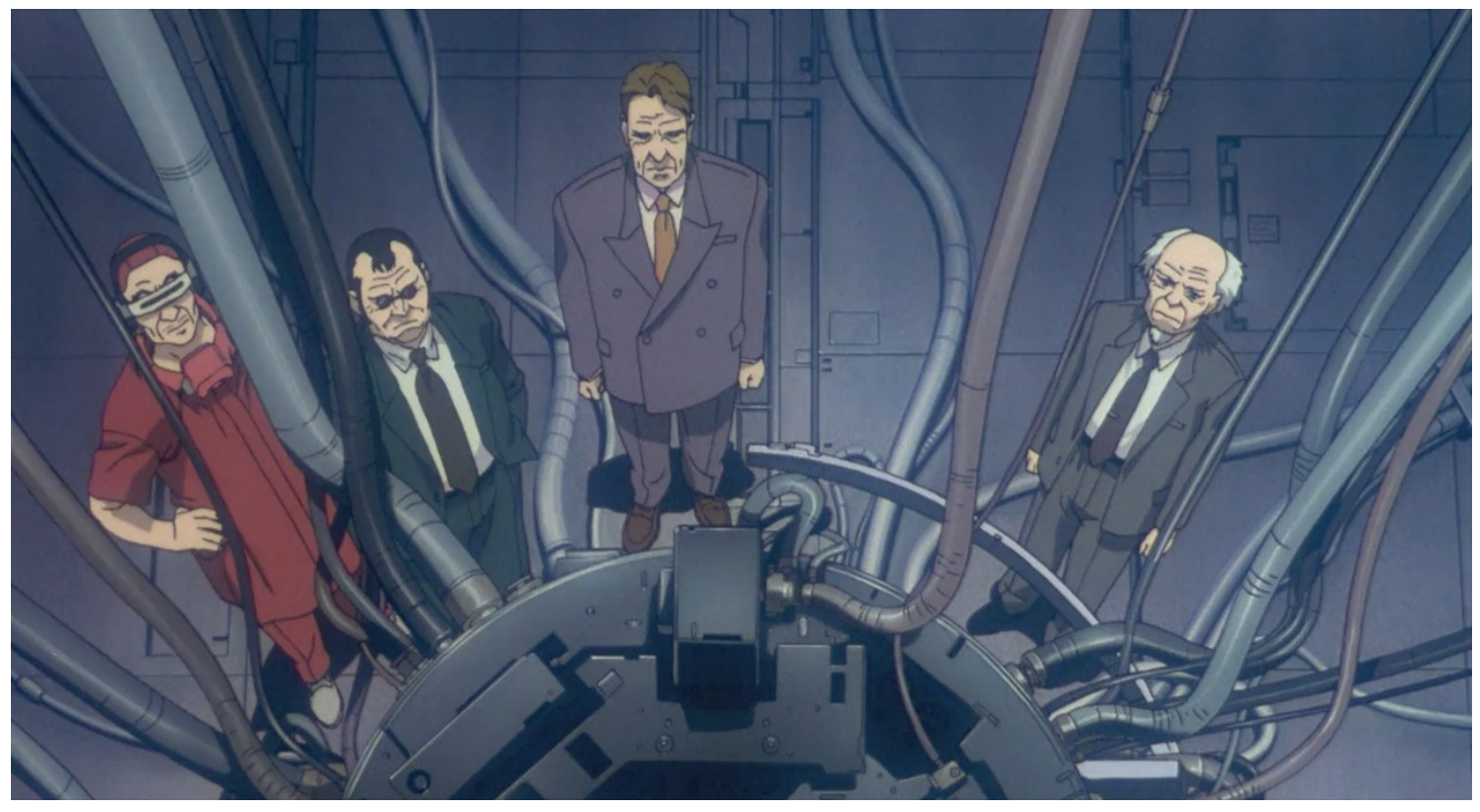

Figure 13: Dr. Willis (center).

If classical rules of iconography or symmetry are followed, Dr. Willis is the central figure among the men.

Left and right of him are two powerful men : one Mr. Nakamura (from the Foreign Ministry) and Aramaki, who heads Section 9.

Also among them (to the far edge) is a technician or engineer (dressed in red), nameless.

The exact identity of Dr. Willis remains unknown here.

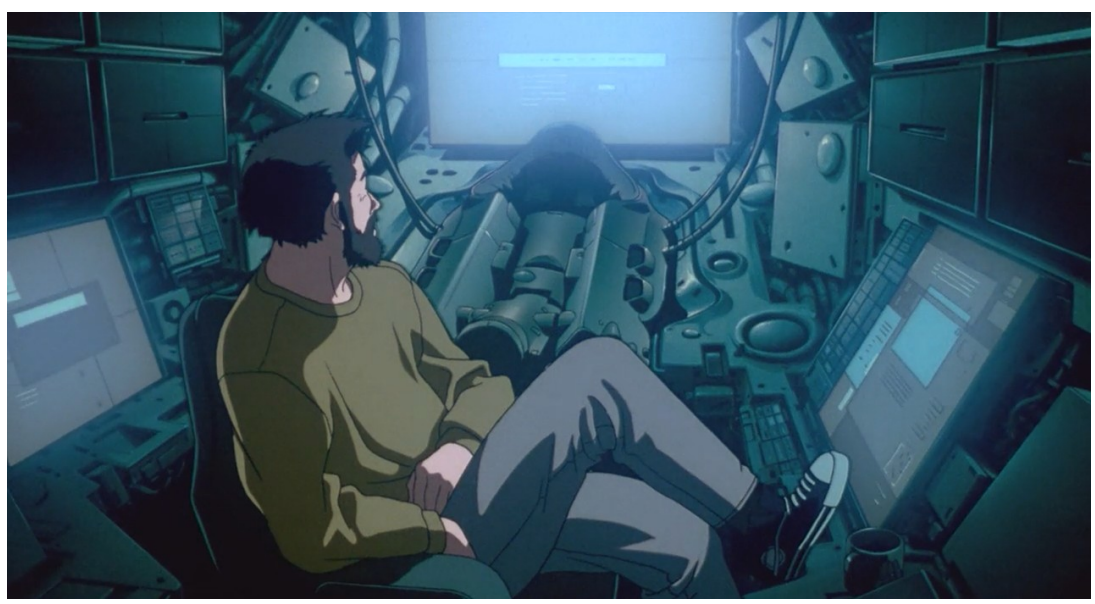

Figure 14: Ishikawa : "I've been divin' around...". 
It is only thanks to the work of a security expert, engineer of some sort (named Ishikawa) that we learn the identity of this nebulous character.

Dr. Willis is the head of this project as well as many other things; as individuals like him tend to be.

Ishikawa - I've been divin' around in the Ministry of Foreign Affairs net. Aramaki - Wait, I'll switch to an encrypted channel.

Ishikawa - Okay now? (...) scoop on that guy who showed up with Nakamura. He's an American : Dr. Willis.

Head of Strategic Research at Neutron Company.

A top researcher in the field of artificial intelligence.

Information that helps make sense of his doings in the previous scene, where his machine parts were additionally exposed (cyborg, robot or else, we don't know).
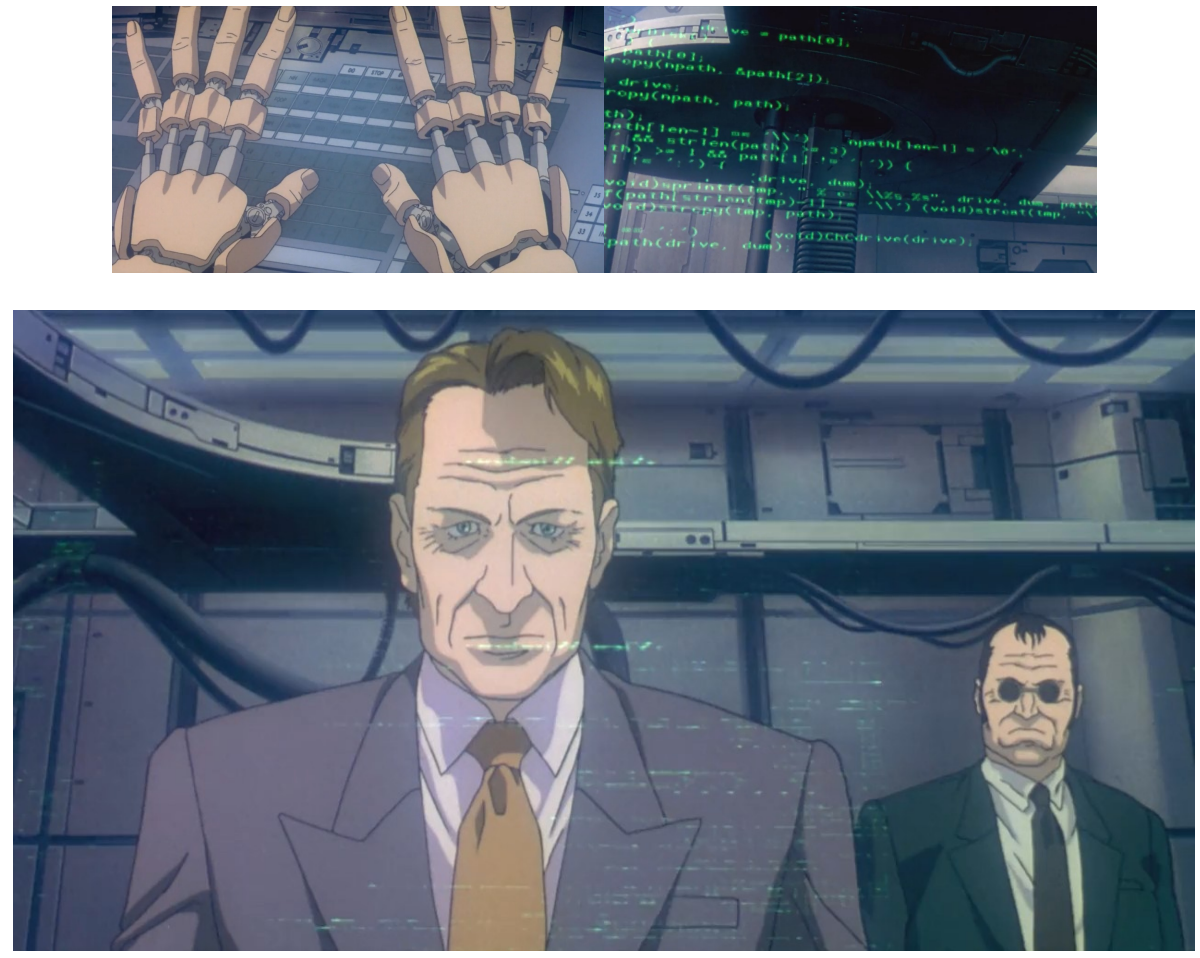

Figure 15: Dr. Willis enters code.

Considering the original aims of the Project (political manipulation), this choice of a computer scientist is fit considering the history of the field of artificial intelligence with regards to (dubious) affiliations ${ }^{15}$.

\footnotetext{
${ }^{15}$ With the private sector among others - which we have pointed out elsewhere. E.g. Hinton (Google), LeCun (Facebook, VP and Chief AI Scientist), Karpathy (Tesla) - and the rest of this peculiar crowd.
} 


\section{Computing and society in video games}

The years from 1993 to 1995 represented some golden moment for video games where themes relating to computing and society were concerned : Shadowrun (1993), Beneath a Steel Sky (1994), System Shock (1994) and the Western version of Snatcher (1994 EU; 1995 NA) being released in close succession of each other.

They all had been preceded by Snatcher in 1988 .

In the search for antecedents - an endless and almost futile quest - we included Metroid, with reservations.

We concentrated on the earliest such cultural products.

They had for (often common) themes :

- the merger of humans and machines (variously called "cyborgs" or "androids" or some other - roid nomenclature)

- a fundamental re-examination of gender and sex (based on these changes)

- artificial intelligence and robotics

- very large companies and governments

Many, no doubt, have since appeared (and will appear) with the same themes and preocupations.

\subsection{Metroid (1986)}

Metroid, a platform ('jump and run') game, had story and themes that comparatively drew more from space epics (opposed by cyberpunk) ${ }^{16}$.

"fortress planet", "galaxy federal police", background of stars, etc.

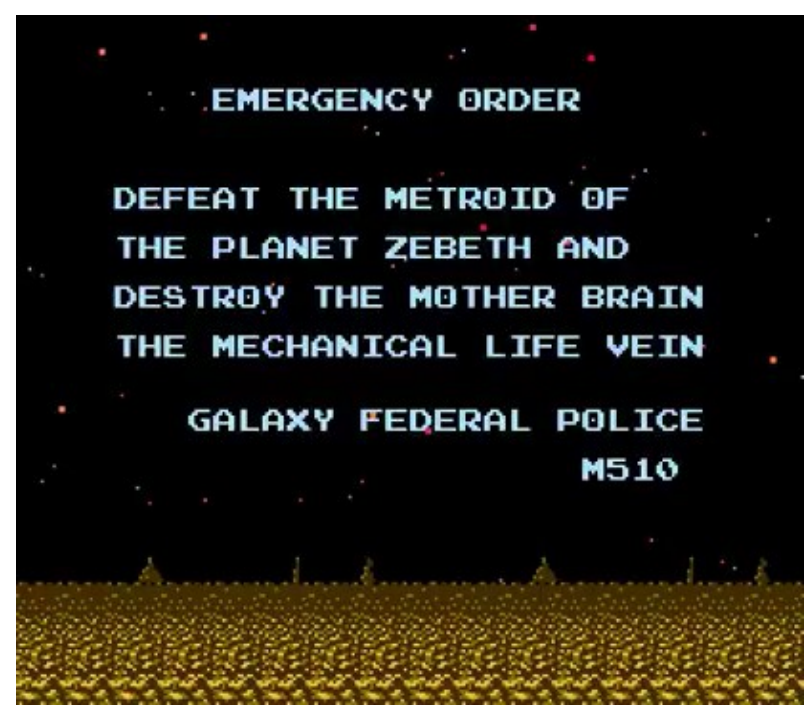

Figure 16: The entire script for $99 \%$ of Metroid (1986).

\footnotetext{
${ }^{16}$ e.g. Asimov and Herbert in literature, Star Wars and Star Trek in cinema. Although Metroid itself seemed to draw more from Alien (1979) specifically.
} 


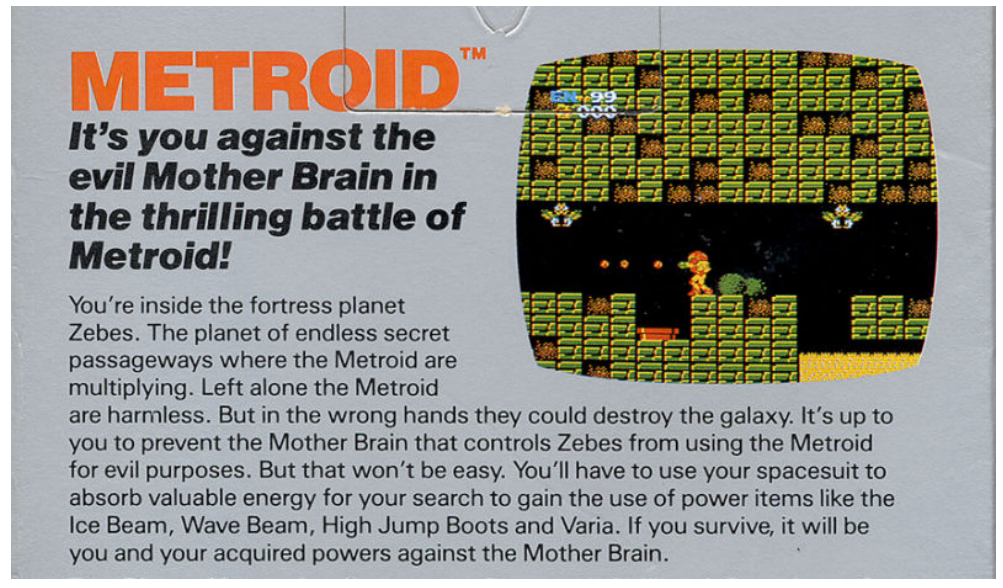

Figure 17: Box of Metroid (C 1987 Nintendo America).

Protagonist Samus Aran has body enhancements (in the form of an armor or rather "spacesuit", and weapons).

Their gender is object of mystery.

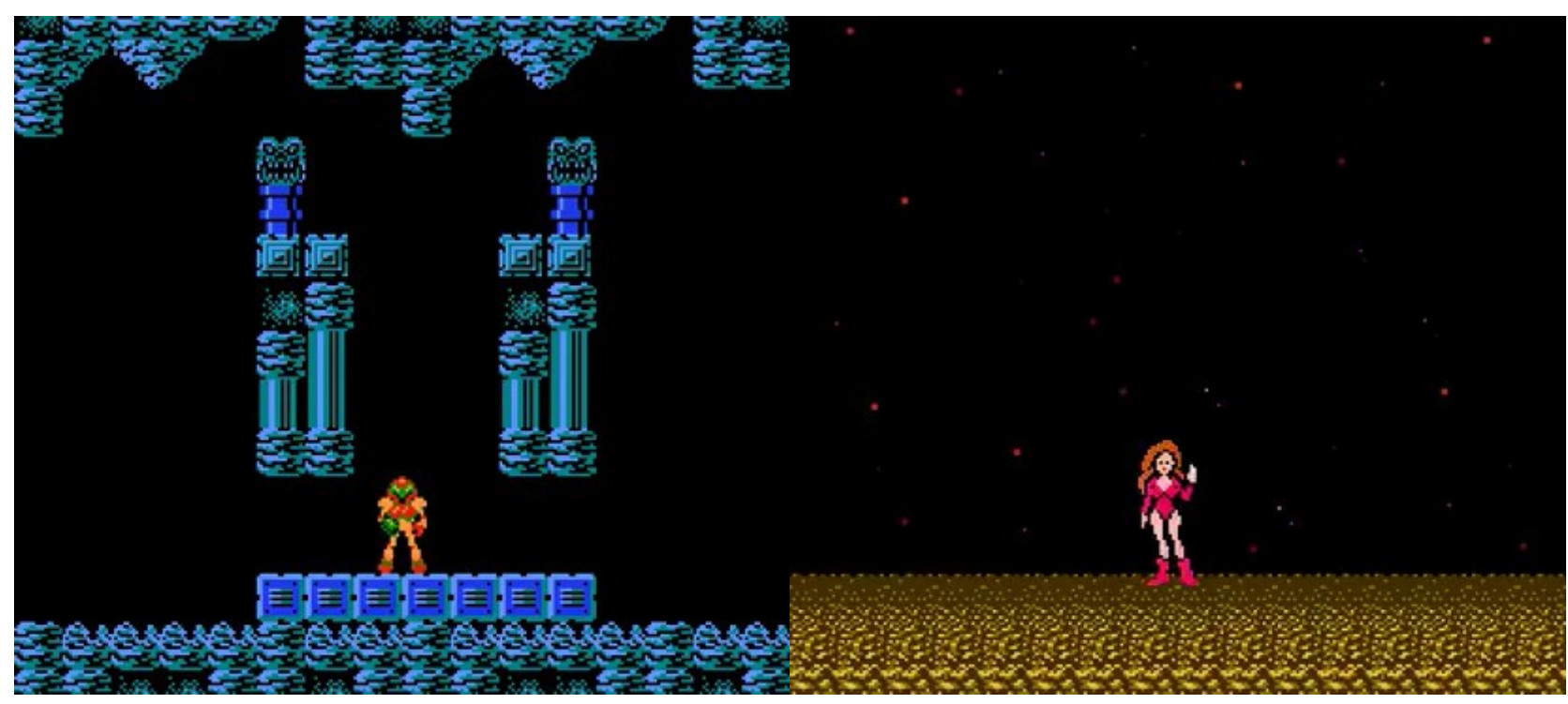

Figure 18: Samus (she/they/he/*) at the beginning and end of Metroid.

\subsection{Snatcher $(1988 / 1994)$}

"This story is dedicated to all those cyberpunks who fight against injustice and corruption every day of their lives." - epitaph 
Author Hideo Kojima ${ }^{1718}$ created with Snatcher, published $1988^{19}$, what is likely the first, most ambitious and mature project of its kind, a video game - a "cyberpunk adventure" (as its cover stated) - that contained the themes explored in literature and film in the decades before:

"[S]cience fiction dealing with future urban societies dominated by computer technology.", according to one definition of cyberpunk. ${ }^{20}$

Examples of which range from novels like Philip K. Dick's Do Androids Dream of Electric Sheep? (1968) to movies like Blade Runner (1982), adapted from the former, through Gibson's short story "Hologram Rose" (1977) and later novel Neuromancer (1984).

Androids are responsible for "humanity's greatest crisis"; a "life form", "part organic, part machine".

"[T]hey can sweat and even bleed" (dotted with an "artificial skin").

"They are almost indistinguishable from" humans.

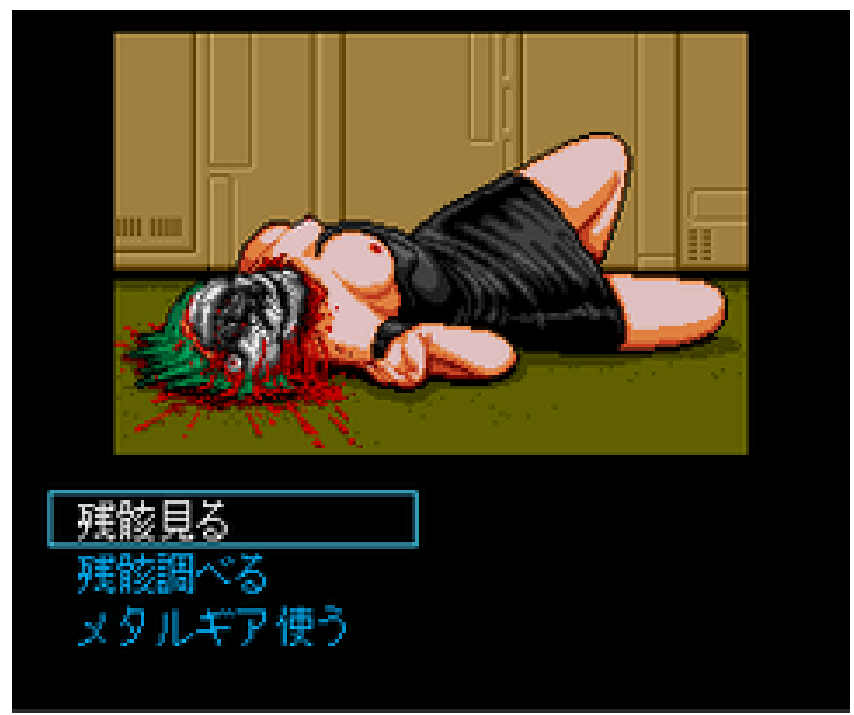

Figure 19: An android in Snatcher.

These androids are called "snatcher(s)" because of their "killing humans and taking their place".

"Its purpose and origin are unknown" - the (voice-over) introduction continues.

In Dec. $2047^{21}$ new Kobe ("Neo Kobe City"), protagonist Gillian Seed

${ }^{17}$ Best known for the acclaimed Metal Gear series.

${ }^{18} \mathrm{He}$ is credited as "creator" in the English-language version.

${ }^{19}$ A Western version appeared in 1994.

${ }^{20}$ https://www.merriam-webster.com/dictionary/cyberpunk

${ }^{21}$ This narrative choice, of December or Christmas, is shared with e.g. 12 Monkeys (1995). 
becomes a new member of JUNKER ${ }^{22}$, "a special task force put together to combat the 'bioroid SNATCHER' menace", "overseen by government intelligence agencies".

To help him he is given a robot : 'metal gear' (a 'personal assistant').

A member of this group/organization is named Gibson.

A quest to regain "lost memories" and "to remember [the] past." ("our past").
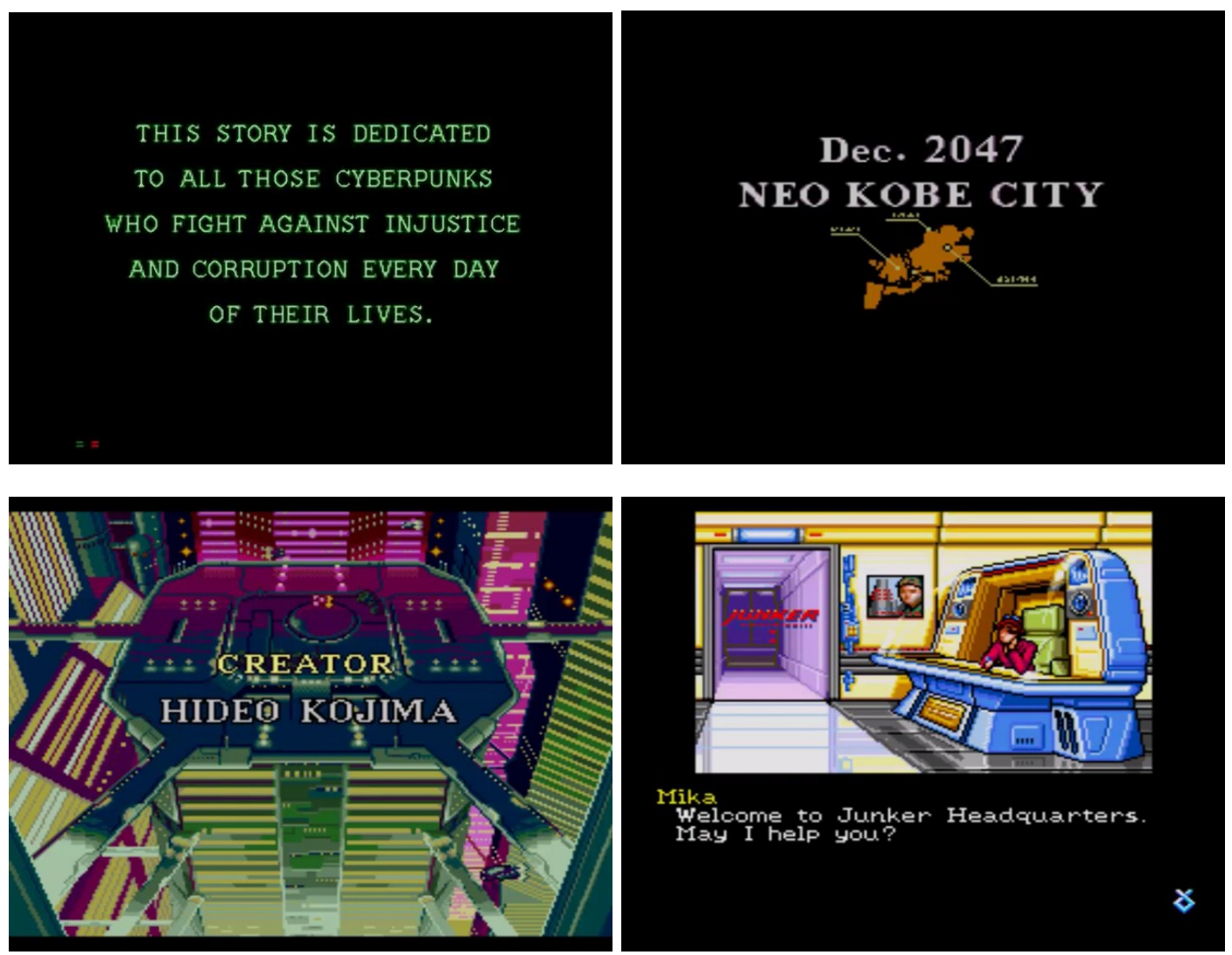

Figure 20: Opening scenes of Snatcher.

\footnotetext{
${ }^{22}$ Pronounced junk-er. (A likely poor choice in translation/localization considering the (historical) meanings of this word.)
} 


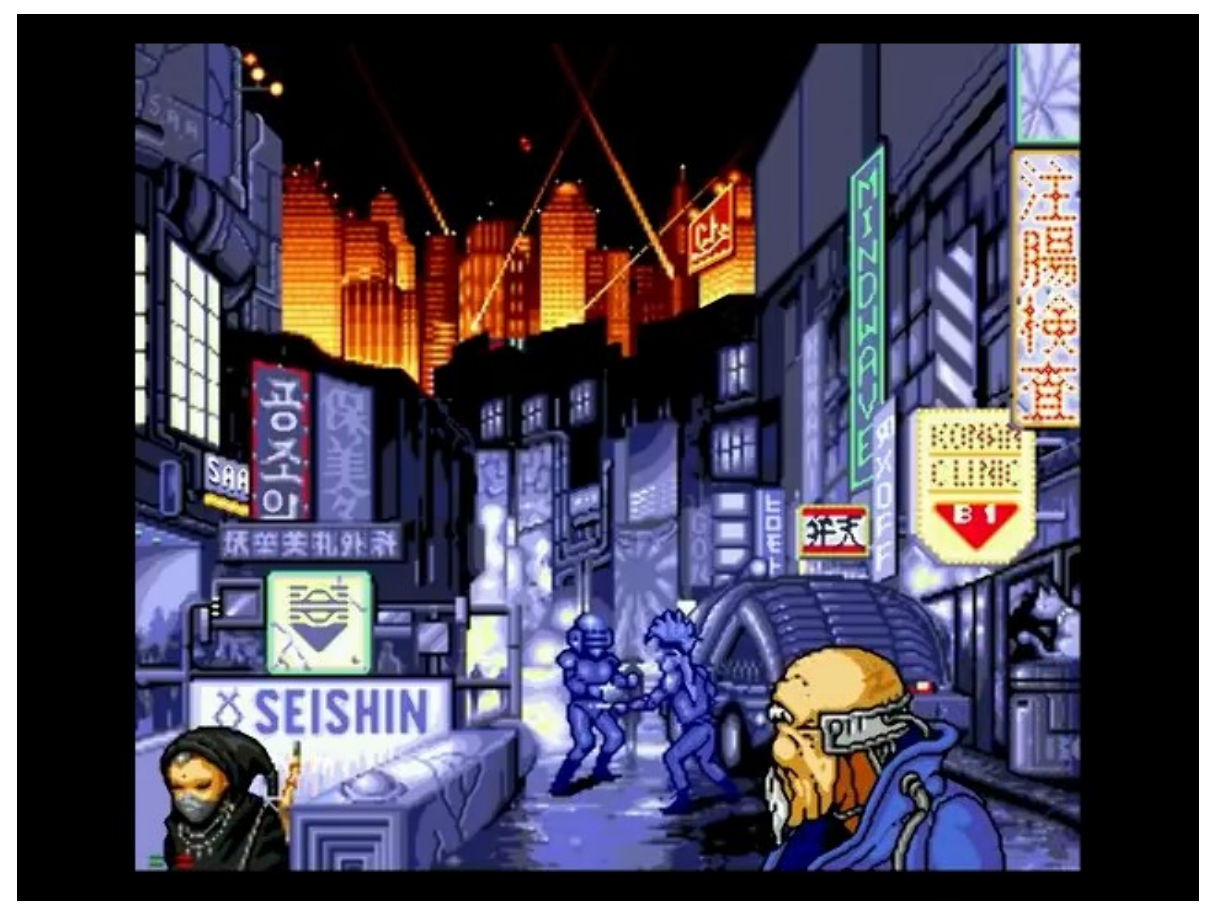

Figure 21: (Further into) Snatcher.

\subsection{From Shadowrun to System Shock}
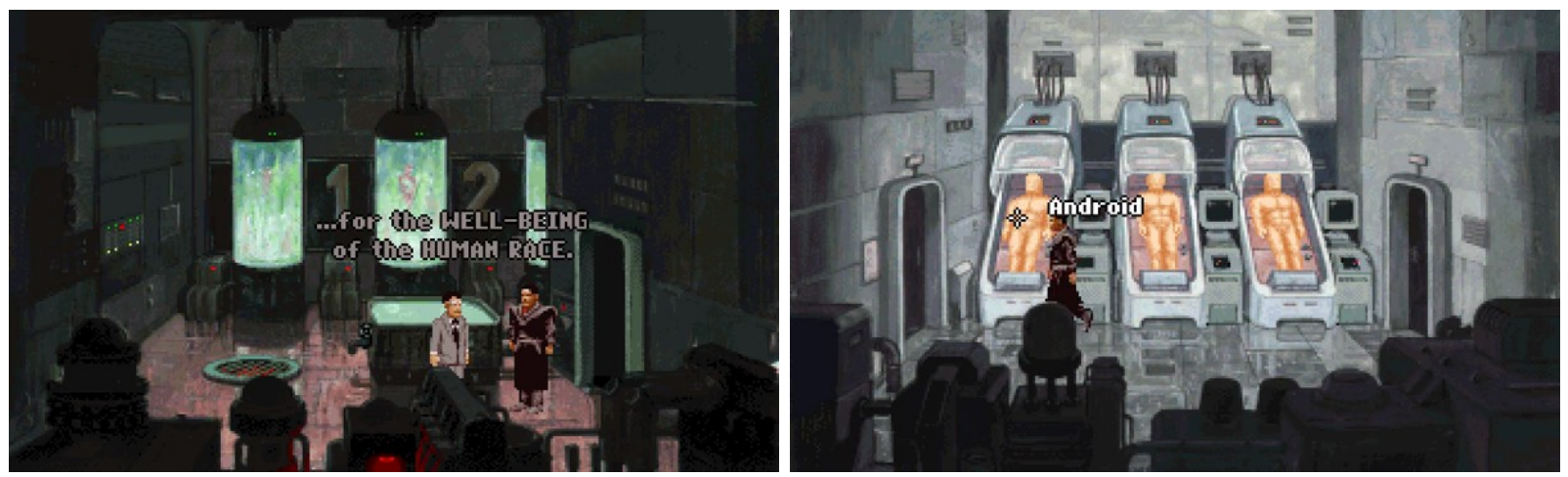

Figure 22: Beneath a Steel Sky (1994).

In Beneath a Steel Sky, a British production ${ }^{23}$, a (central) system called LINC forms society's backbone. ${ }^{23}$ Fact exceptional enough to be noted in an industry (historically) dominated by Japanese
and U.S.-American companies. 
Its ending sees the protagonist ask, and receive for answer :

- You're not HUMAN, are you?

- Now WHATEVER gave you that idea?

I'm OBVIOUSLY not a MACHINE...

Or an ANIMAL...

And, that this technology and whatever plans their makers may have are "for the well-being of the human race." (announcing simultaneously otherwise). An Oppenheimer-like figure.
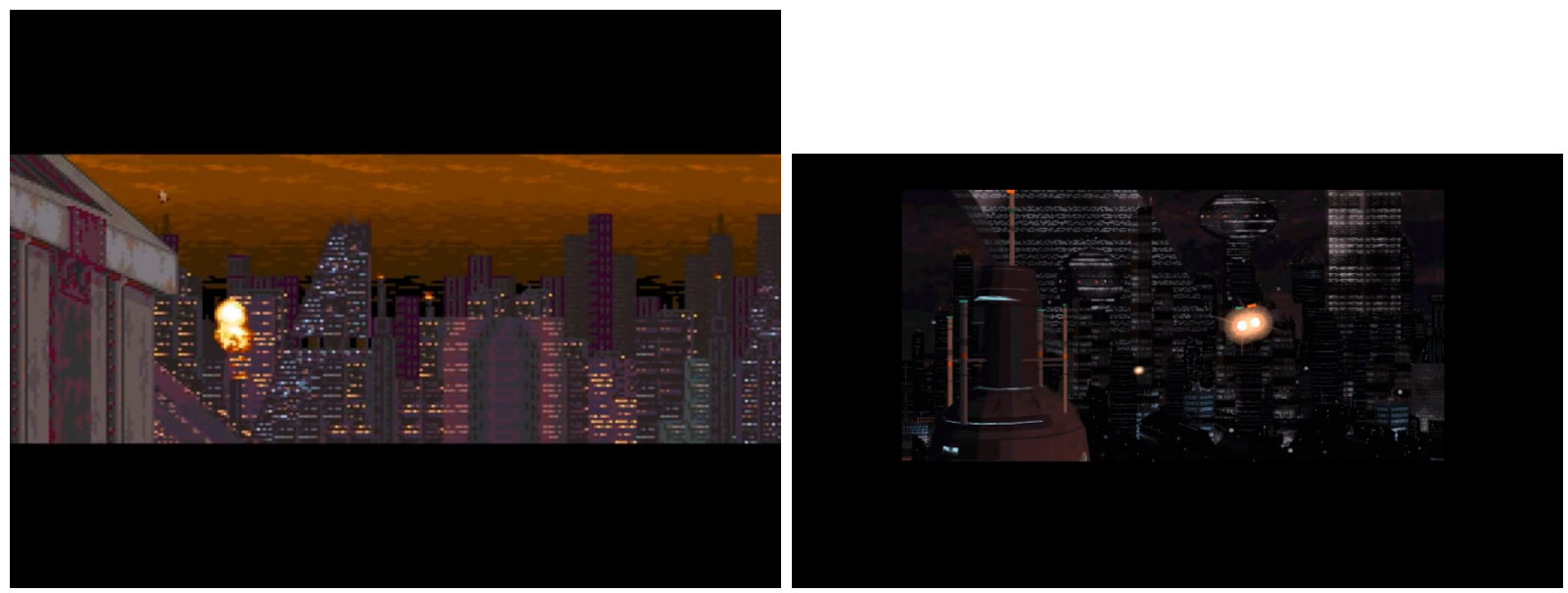

(b) New Atlanta

Figure 23: Shadowrun and System Shock.

Shadownrun opened with the following introduction ${ }^{24}$ :

THE YEAR IS 2050

And the megaplexes are monsters casting long shadows.

When you become a shadowrunner, that's where you live...

in the cracks between the giant corporate structures.

The megacorps are powerful in a time when power means information...

...computer information flowing in the global network called the MATRIX.

Here, not governments, but large companies - called "megacorps" are presented as the biggest threat (to humanity).

This contrasts with contemporary debates, which lead by few but influential individuals and institutions (of Libertarian backgrounds mostly ${ }^{25}$ ),

\footnotetext{
${ }^{24}$ Note this game was released half a decade before The Matrix.

${ }^{25}$ Followed by anarchists.
} 
make governments often the primary, central or sole adversary of human rights, in particular privacy.

A semi-famous computer science $\mathrm{PhD}$ thesis published at the turn of the millennium had for dedication :

"To my parents, advisors, and colleagues, for pushing me to finish this thesis.

And to the US Government, for at least keeping things interesting." (p. 1)

In many more passages, its author goes on about "the government" (with a capital $\mathrm{G}$ ),

"Government is one of the biggest consumers and producers of dossiers of personal information" (p. 15)

but he never seemed to have considered companies.

In that, he is not alone and representative of a certain class of computer scientists and technologists (primarily from the United States).

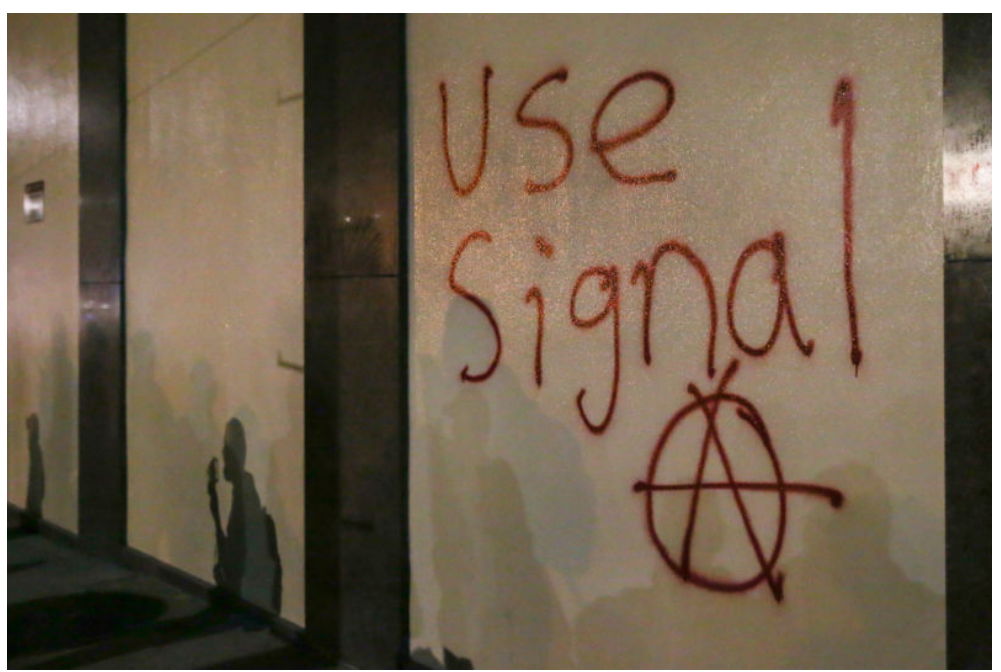

Figure 24: A random graffiti in Berkeley, California.

Libertarianism and anarchism : two faces of the same coin.

To the ideology of the father, corresponds that of the son; and to that of the bourgeois wife her former rebellious self.

In the end, all are faithful to their class of origin. 